Pacific Journal of Mathematics

A SPECTRAL MAPPING THEOREM FOR LOCALE 


\title{
A SPECTRAL MAPPING THEOREM FOR LOCALLY COMPACT GROUPS OF OPERATORS
}

\author{
Claudio D'Antoni, Roberto Longo and \\ LASZLO ZSIDO
}

\begin{abstract}
If $U$ is a suitably continuous representation of a locally compact abelian group $G$ by means of isometries on a Banach space $X, \mu \rightarrow U(\mu)$ its extension to a representation of the convolution algebra $M(G)$ and $\operatorname{sp}(U)$ the spectrum of $U$, then the spectrum of $U(\mu)$ is not always equal to $\hat{\mu}(\operatorname{sp}(U))^{-}$, but it is so if the continuous part of $\mu$ is absolutely continuous.
\end{abstract}

1. Introduction. To be more explicit, given a representation $U$ of $G$ as above one forms a representation of $M(G)$, the Banach algebra of bounded regular measures on $G$, given by

$$
U: \mu \in M(G) \longrightarrow U(\mu)=\int U(g) d \mu(g) \in B(X) .
$$

In particular, if $G=R, U(\mu)$ can be interpreted in a more classical way as a function of the infinitesimal generator $D=\left.i(d / d g) U(g)\right|_{g=0}$ and denoted by $\hat{\mu}(D)$, where $\hat{\mu}$ is the Fourier transform of $\mu$. Notice that in this case $\sigma(D)=\operatorname{sp}(U)[5,9]$, where $\sigma$ is the usual spectrum of the linear operator $D$ and $\operatorname{sp}(U)$ is the spectrum of the representation $U$ (see [2]).

Thus it is natural to study how far this functional calculus can be extended and a spectral mapping theorem holds. The setting of our study will be the algebra of local multipliers of $L^{1}(G)$.

If $\mu$ is a Dirac measure, A. Connes [3] proved that

$$
\sigma(U(\mu))=\hat{\mu}(\operatorname{sp}(U))^{-} .
$$

Even if such a result does not always extend (we shall exhibit counterexamples) we prove it for the class of measures whose continuous part belongs to $L^{1}(G)$.

2. Statement of the main result. Let $G$ be a locally compact abelian group; by a representation $U$ of $G$ on a Banach space $X$ we mean a pointwise $\sigma\left(X, X^{*}\right)$-continuous homomorphism of $G$ into the group of $\sigma\left(X, X^{*}\right)$-continuous isometries of $X$, where $X^{*}$ is the dual of $X$ or $X$ is the dual of $X^{*}$. The case of bounded representations reduces to this one.

Let $M(G)$ be the Banach algebra of all bounded regular measures on $G$. Given any algebra $L^{1}(G) \subset M \subset M(G)$, we can form the representation of $M$ induced by $U$ : 


$$
U(\mu)=\int U(g) d \mu(g), \quad \mu \in M .
$$

The spectrum of $U$ is a closed subset of the dual $\hat{G}$ of $G$ defined by means of Fourier transforms (see [2] for this and related matter)

$$
\operatorname{sp}(U)=\{p \in \hat{G} / U(\mu)=0 \Longrightarrow \hat{\mu}(p)=0, \quad \mu \in M\}
$$

and it does not depend on $M$.

The following lemma will be later generalized.

Lemma 1. For every $\mu \in M(G)$ we have $\sigma(U(\mu)) \supset \hat{\mu}(\operatorname{sp}(U))^{-}$, where $\sigma$ denotes the usual spectrum in $B(X)$.

Proof. We have to show that if $p \in \operatorname{sp}(U)$, then $\hat{\mu}(p) \in \sigma(U(\mu))$. Indeed if $p \in \operatorname{sp}(U)$ there exists a net $\left\{x_{i}\right\} \subset X,\left\|x_{i}\right\|=1$, such that $\left\|U(g) x_{i}-(p, g) x_{i}\right\| \rightarrow 0$ uniformly for $g$ varying in a compact subset $K \subset G$. Let $\varepsilon>0$ and $K \subset G$ such that $|\mu|(G \backslash K)<\varepsilon / 4$ and choose $x \in\left\{x_{i}\right\}$ such that

$$
\left\|\int_{K} U(g) d \mu(g) x-\int_{K}(p, g) d \mu(g) x\right\|<\varepsilon / 2
$$

then

$$
\begin{aligned}
\left\|U(\mu) x_{i}-\hat{\mu}(p) x_{i}\right\| \leqq & \left\|\int_{K} U(g) d \mu(g) x_{i}-\int_{K}(p, g) d \mu(g) x_{i}\right\| \\
& +\left\|\int_{G \backslash K} U(g) d \mu(g) x_{i}-\int_{G \backslash K}(p, g) d \mu(g) x_{i}\right\|<\varepsilon
\end{aligned}
$$

that entails the lemma.

REMARK 1. The reverse inclusion in the above lemma is not true for every $\mu \in M(G)$ : in fact, if $G$ is not discrete, $X=L^{1}(G)$ and $U(g)$ is the translation by $g$, then, due to asymmetry of $M(G)$, there exists $\mu_{0} \in M(G)$ such that $\sigma\left(U\left(\mu_{0}\right)\right) \neq \hat{\mu}_{0}(\widehat{G})-$ (see [11]). By the same reasoning we can give a counterexample for automorphism groups of factors. Indeed let $\alpha=U^{\prime}$ be the transposed action on $L^{\infty}(G)$; we have $\hat{\mu}_{0}(\hat{G}) \varsubsetneqq \sigma\left(U\left(\mu_{0}\right)\right)=\sigma\left(U^{\prime}\left(\mu_{0}\right)\right)=\sigma\left(\alpha\left(\mu_{0}\right)\right)$. If $\tilde{\alpha}$ is an extension of $\alpha$ to $B\left(L^{2}(G)\right)$, then $\hat{\mu}_{0}(\widehat{G}) \varsubsetneqq \sigma\left(\alpha\left(\mu_{0}\right)\right) \subset \sigma\left(\tilde{\alpha}\left(\mu_{0}\right)\right)$.

THEOREM 1. For every $\mu \in M(G)$ whose continuous part belongs to $L^{1}(G)$ we have $\sigma(U(\mu))=\hat{\mu}(\operatorname{sp}(U))^{-}$.

The proof of this theorem requires some lemmas.

3. Identification of spectra. Let $M$ be a subset of $M(G)$; by 
$A(M)$ we denote the closure in $B(X)$ of $\{U(\mu): \mu \in M\}$. We recall the following identification of $\operatorname{sp}(U)$. [3, Prop. 2.3.7].

Proposition 2. The map $p \in \operatorname{sp}(U) \rightarrow j(p) \in \sigma\left(A\left(L^{1}(G)\right)\right)$ defined by

$$
j(p)(U(f))=\hat{f}(p) \quad f \in L^{1}(G)
$$

establishes an homeomorphism of $\mathrm{sp}(U)$ onto the spectrum of $A\left(L^{1}(G)\right)$.

If $M$ is a Banach algebra and $L^{1}(G) \subset M \subset M(G)$ we split $\sigma(A(M))$ into two disjoint sets $\sigma(A(M))=H(M) \cup \Omega(M)$ where $H(M)=$ $\left\{\chi \in \sigma(A(M)) / \chi \uparrow A\left(L^{1}(G)\right)=0\right\}$ and $\Omega(M)$ is the complementary subset.

LemmA 3. (i) The map $\chi \in \Omega(M) \rightarrow \chi \uparrow A\left(L^{1}(G)\right)$ is an homeomorphism of $\Omega(M)$ onto the spectrum of $A\left(L^{1}(G)\right)$.

(ii) Let $\pi: A(M) \rightarrow A(M) / A\left(L^{1}(G)\right)$ be the quotient map. Then $\varphi \in \sigma\left(A(M) / A\left(L^{1}(G)\right)\right) \rightarrow \varphi \cdot \pi$ is an homeomorphism of $\sigma\left(A(M) / A\left(L^{1}(G)\right)\right)$ onto $H(M)$.

Proof. (i) Let $\chi_{0} \in \sigma\left(A\left(L^{1}(G)\right)\right)$. By Proposition 2 there exists $p \in \operatorname{sp}(U)$ such that $j(p)=\chi_{0}$. It is enough to show that $\chi_{0}$ uniquely extends to $\chi \in \sigma(A(M))$ determined by $\chi(U(\mu))=\hat{\mu}(p)$. In fact let $f \in L^{1}(G), \hat{f}(p) \neq 0$. Then $\chi(U(\mu) U(f))=\chi\left(U\left(\mu^{*} f\right)\right), \mu \in M$, thus $\chi(U(\mu)) \hat{f}(p)=\hat{\mu}(p) \hat{f}(p)$ and $\chi(U(\mu))=\hat{\mu}(p)$ for any extension $\chi$ of $\chi_{0}$.

(ii) This fact is known to be valid in more general situations $[10, \S 15]$.

Let $G_{d}$ be the group obtained equipping $G$ with the discrete topology and $U_{d}$ the representation of $G_{d}$ naturally derived by $U$. It follows that $\operatorname{sp}\left(U_{d}\right)=\operatorname{sp}(U)^{-}$[2], where the closure will be always taken in $\hat{G}_{d}$ the Bohr compactification of $\hat{G}$. Proposition 2, with $G=G_{d}$, gives rise to a natural identification of $\operatorname{sp}\left(U_{d}\right)$ with $\sigma\left(A\left(M_{d}(G)\right)\right)$

$$
\begin{aligned}
& p \in \operatorname{sp}\left(U_{d}\right) \longrightarrow j_{d}(p) \in \sigma\left(A\left(M_{d}\right)\right) \\
& j_{d}(p)(U(\mu))=\hat{\mu}(p)
\end{aligned}
$$

where $M_{d}(G)=M\left(G_{d}\right)=L^{1}\left(G_{d}\right)$ is the Banach algebra of discrete measure on $G$ and $\hat{\mu}$ is the Fourier transform of $\mu$ as an element of $L^{1}\left(G_{d}\right)$.

The Banach algebra of measures of interest to us will be

$$
\mathscr{C l}=\left\{\mu \in M(G) / \mu_{c} \in L^{1}(G)\right\}
$$

where $\mu_{c}$ is the continuous part of $\mu$. Let $\mathscr{A}=A(\mathscr{C}), \mathscr{C}=H(\mathscr{C})$ and $\Omega=\Omega(\mathscr{C})$ which is homeomorphic to $\operatorname{sp}(U)$. We define 


$$
\operatorname{sp}_{d}(U)=\left\{p \in \operatorname{sp}\left(U_{d}\right) / \exists \chi \in \mathscr{K} \text { s.t. } j_{d}(p)=\chi \uparrow A\left(M_{d}\right)\right\}
$$

LEMMA 4. If $G$ is nondiscrete $\operatorname{sp}_{d}(U)$ is naturally homeomorphic to $\mathscr{K}$ by the following map:

$$
p \in \operatorname{sp}_{d}(U) \longrightarrow \chi \uparrow A\left(M_{d}\right)=j_{d}(p)
$$

Proof. If $\chi \in \mathscr{H}$ and $\chi \uparrow A\left(M_{d}\right) \neq 0$, then by (3.2) there exists $p \in \operatorname{sp}\left(U_{d}\right)$ such that $\chi(U(\mu))=\hat{\mu}(p)$ for every $\mu \in M\left(G_{d}\right)$. Obviously $j_{d}(p)=\chi \uparrow A\left(M_{d}\right)$, therefore $p \in \mathrm{sp}_{d}(U)$ by definition, and the map in (3.4) is continuous. On the other hand for any $p \in \operatorname{sp}_{d}(U), j_{d}(p)$ extends to $\chi \in \mathscr{H}$ by $\chi(U(\mu))=\hat{\mu}(p)$ establishing a continuous inverse of the above map.

4. Topological lemmas, Let $G$ and $G_{d}$ be as above. We shall identify $M_{d}(G)$ and $L^{1}\left(G_{d}\right)$. No confusion will arise since, if $\mu \in L^{1}\left(G_{d}\right)$, then $\hat{\mu} \uparrow \widehat{G}$ is the Fourier transform of $\mu$ as an element of $M_{d}(G)$.

LEMMA 5. For each compact subset $K$ in $\operatorname{sp}(U)$ we have

$$
\operatorname{sp}_{d}(U) \subset \overline{\overline{\operatorname{sp}(U) \backslash K}} \text {. }
$$

Proof. Let us assume that there is a $p \in \operatorname{sp}_{d}(U)$ such that $p$ does not belong to $\overline{\overline{\operatorname{sp}(U)} \backslash K}$. This will lead to a contradiction. Indeed if the thesis is not fulfilled there is an open set $V$ in $\widehat{G}_{d}$ such that $V$ contains $p \in \operatorname{sp}_{d}(U)$ and $\left.V \cap \overline{(\operatorname{sp}(U)} \backslash K\right)=\varnothing$. This means that $V \cap \overline{\operatorname{sp}(U)} \subset K$. Let $\mu$ be a measure in $L^{1}\left(G_{d}\right)=M\left(G_{d}\right)$ such that $\operatorname{supp}(\hat{\mu}) \subset V, \hat{\mu}(p)=1$. Therefore $\operatorname{supp}(\hat{\mu}) \cap \operatorname{sp}(U) \subset K$. As $K$ is compact there exists $f \in L^{1}(G)$ such that $U(\mu)=U(f)$. If $\chi \in H$ is the character corresponding to $p \in \operatorname{sp}_{d}(U)$ as in $(2.4)$, then $\chi(U(f))=$ 0 and $0=\chi(U(f))=\chi(U(\mu))=1$.

The following lemma can be proved by elementary consideration.

LEMMA 6. Let $K$ be a compact set, $F$ a closed set with $K \subset F \subset$ $\hat{G}$. Then $\overline{\bar{F} \backslash K} \subset \overline{F \backslash K}$, where, as always, the closure are taken in $\hat{G}_{d}$. In particular for any compact set $K \subset \operatorname{sp}(U)$, we have $\operatorname{sp}_{d}(U) \subset$ $\overline{\operatorname{sp}(U) \backslash K}$.

5. Proof of Theorem 1. Let $\mu \in M(G)$ be such that $\mu=\mu_{c}+$ $\mu_{d}$ with $\mu_{c} \in L^{1}(G)$ and $\mu_{d} \in M_{d}(G)$. We have to show that $\hat{\mu}(\operatorname{sp}(U))^{-} \supset$ $\sigma\left(U(\mu)\right.$ ). Since $\sigma(U(\mu)) \subset \sigma_{A}\left(U(\mu)\right.$ ) (where $\sigma_{A}$ is the spectrum relative to $\mathscr{A})$, it is sufficient to prove that

$$
\hat{\mu}(\operatorname{sp}(U))^{-} \supset \sigma_{A}(U(\mu))
$$


It is enough to show that if $0 \notin \hat{\mu}(\operatorname{sp}(U))^{-}$then $U(\mu)$ is invertible in \&. That is $\chi(U(\mu)) \neq 0$ for every $\chi \in \sigma(\mathscr{A})$. Assume $0 \notin \hat{\mu}(\operatorname{sp}(U))^{-}$ and let $\varepsilon_{0}>0$ be such that

$$
|\widehat{\mu}(p)| \geqq \varepsilon_{0} \quad p \in \operatorname{sp}(U) .
$$

If $\chi \in \sigma(\mathscr{A})$ there are two possibilities, $\chi \in \Omega$ or $\chi \in \mathscr{C}$ (see 3).

(a) If $\chi \in \Omega$ then there exists $p \in \operatorname{sp}(U)$ such that $\chi(U(\mu))=\hat{\mu}(p)$ for every $\mu \in \mathscr{A}$, therefore

$$
|\chi(U(\mu))|=|\hat{\mu}(p)| \geqq \varepsilon_{0}>0 .
$$

(b) If $\chi \in \mathscr{Y}$ let $p_{0} \in \mathrm{sp}_{d}(U)$ be such that (cf Lemma 4)

$$
\chi(U(\mu))=\hat{\mu}_{d}\left(p_{0}\right)
$$

where $\mu_{d}$ is considered as an element of $L^{1}\left(G_{d}\right)$. Let

$$
K=\left\{p \in \operatorname{sp}(U) /\left|\hat{\mu}_{c}(p)\right| \geqq \varepsilon_{0} / 2\right\},
$$

then, since $\hat{\mu}_{c}$ vanishes at infinity, $K$ is compact. Since $\left|\hat{\mu}_{c}(p)+\hat{\mu}_{d}(p)\right| \geqq \varepsilon_{0}$ for $p \in \operatorname{sp}(U)$, we have $\left|\hat{\mu}_{d}(p)\right| \geqq \varepsilon_{0} / 2$ for $p \in$ $\operatorname{sp}(U) \backslash K$. Since $\hat{\mu}_{d}$ is continuous on $\hat{G}_{d}$ we have $\left|\hat{\mu}_{d}(p)\right| \geqq \varepsilon_{0} / 2$ for every $p$ in $\overline{\operatorname{sp}(U) \backslash K} \supset \operatorname{sp}_{d}(U)$, and therefore, by (4.2), $|\chi(U(\mu))|=$ $\left|\hat{\mu}_{d}\left(p_{0}\right)\right| \geqq \varepsilon_{0} / 2>0$ because $p_{0} \in \operatorname{sp}_{d}(U)$.

6. Functional calculus for local multipliers. We consider now an involutive algebra $\mathfrak{M}=\mathfrak{M}(G, U)$ of local multipliers for $L^{1}(G)$, namely $\hat{F} \in \mathfrak{M}$ iff $\hat{F}$ is a complex function defined on a neighborhood of $\operatorname{sp}(U)$ and locally belongs to $L^{1}(G)$ at every point $p \in$ $\operatorname{sp}(U)$.

Let $D_{0}(U)$ be the union of the spectral subspaces $X(E, U)$ of $U$ corresponding to compact subsets $E$ of $G$ (cf $[2,12]$ )

$$
D_{0}(U)=\bigcup_{E} X(E, U), \quad E \text { compact subset of } G \text {. }
$$

Owing to the regularity of $L^{1}(G)$, we can define, for every $\hat{F} \in \mathfrak{M}$, the linear operator $U(F): D_{0}(U) \subset X \rightarrow X$ by

$$
U(F) x=U(f) x, \quad x \in X(E, U), \quad E \text { compact }
$$

where $f$ is an arbitrary element of $L^{1}(G)$ such that $\hat{f}$ is equal to $\hat{F}$ on a neighborhood of $E$. In such a way $\{U(F), \hat{F} \in \mathfrak{M}\}$ becomes an involutive algebra of operators of $X$ on the common dense invariant domain $D_{0}(U)$ (with involution given by $U(F) \rightarrow U(\bar{F})$ ). Every $U(F)$ is closable because $D\left(U(F)^{\prime}\right)$, the domain of the transposed of $U(F)$, is dense in $X^{*}$, as shown in the following lemma. Note that $U^{\prime}$, the transposed representation of $U$, is $\sigma\left(X^{*}, X\right)$-continuous; if $\mu \in$ 
$M(G), U^{\prime}(\mu)$ is a bounded linear operator of $X^{*}$ and $(U(\mu))^{\prime}=U^{\prime}(\mu)$. Define $D_{0}\left(U^{\prime}\right) \subset X^{*}$ as in (6.1).

Lemma 7. $\quad D_{0}\left(U^{\prime}\right)$ is contained in $D\left(U(F)^{\prime}\right)$ for every $\hat{F} \in \mathfrak{M}$.

Proof. Fix a compact $E \subset \hat{G}$ and $\varphi \in X^{*}\left(E, U^{\prime}\right)$. If $x \in D_{0}(U)$ there exists $K$ compact $K \subset \widehat{G}$ such that $x \in X(K, U)$. Let $f \in L^{1}(G)$ such that $\hat{f}(p)=\hat{F}(p)$ if $p$ belongs to a neighborhood of $E \cup K$; we have

$$
(U(F) x, \varphi)=(U(f) x, \varphi)=\left(x, U(f)^{\prime} \varphi\right)=\left(x, U^{\prime}(f) \varphi\right)=\left(x, U^{\prime}(F) \varphi\right)
$$

that shows $U(F)^{\prime} \supset U^{\prime}(F)$.

We recall that if $T$ is a linear operator on a Banach space $X$, the extended spectrum $\sum(T)$ is defined as the set of the singularities of the resolvent of $T$ in $C \cup\{\infty\}$.

Lemma 8. For every $\hat{F} \in \mathfrak{M}$ we have $\hat{F}(\operatorname{sp}(U))^{-} \subset \sum(U(F))$.

Proof. As $\sum(U(F))$ is closed, it is enough to prove that $\sum(U(F)) \supset \hat{F}(\operatorname{sp}(U))$. To show this, we consider the representation $U^{E}: g \in G \rightarrow U(g) \uparrow X(E, U)$ obtained by reducing $U$ to the spectral subspace relative to $E \subset \widehat{G}$. Let $E \subset \widehat{G}$ be a compact set and $f \in L^{1}(G)$ such that $\hat{f}=\hat{F}$ on a neighborhood of $E$, so that $U(F) \uparrow X(E, U)=$ $U(f) \uparrow X(E, U)=U^{E}(f)$. Owing to the regularity of $L^{1}(G)$ we have $\operatorname{sp}\left(U^{E}\right) \subset \operatorname{sp}(U) \cap E$, hence

$$
\sum(U(F)) \supset \sum(U(F)) \uparrow X(E, U)=\sum\left(U^{E}(f)\right)=\hat{f}\left(\operatorname{sp}\left(U^{E}\right)\right)=\hat{F}\left(\operatorname{sp}\left(U^{E}\right)\right.
$$

where the second equality is justified by Theorem 1 . Since

$$
\operatorname{sp}(U)=\bigcup_{E} \operatorname{sp}\left(U^{E}\right), \quad E \text { compact subset of } G,
$$

the lemma is proved.

The reverse inclusion in the above lemma cannot be proved for every bounded $\hat{F}$.

Proposition 9. Let $\hat{F}$ be a bounded continuous function in $\mathfrak{M}$ which is not Fourier transform of a measure of $M(G)$. If $U$ is the representation of $G$ on $L^{1}(G)$ by translations given by $(U(g) f)(h)=$ $f\left(g^{-1} h\right), f \in L^{1}(G)$ then $\Sigma(U(F)) \supsetneq \hat{F}(\operatorname{sp}(U)$.)

Proof. We shall derive from our hypotheses that $\sum(U(F))$ cannot be compact. Assuming the contrary there exists a regular 
closed Jordan curve $\Gamma$ containing $\sum(U(F))$ in the interior $\stackrel{\circ}{\Gamma}$. Let $P=(-1 / 2 \pi i) \oint_{\Gamma}(U(F)-\lambda)^{-1} d \lambda . \quad P$ is a projection of $B\left(L^{1}(G)\right)$ that commutes with $U(g), g \in G$ and decomposes $U(F)$ according to $U(F)=$ $U(F) P+U(F)(I-P)$. We have $\sum(U(F) \uparrow P X)=\sum(U(F)) \cap \stackrel{\Gamma}{\Gamma}=$ $\sum(U(F)), \sum(U(F) \uparrow(I-P) X)=\sum(U(F)) \cap(C \backslash \stackrel{\Gamma}{)})=\varnothing$. As $U(F) P$ is bounded and commutes with $U(g), g \in G, U(F) P$ is a multiplier of $L^{1}(G)$ [11]. Therefore $U(F)(I-P)=U(F)-U(F) P$ is a local multiplier. As

$$
\sum(U(F)(I-P)) \subset\{0\},
$$

by Lemma 8 we have that $U(F)(I-P)$ is a multiplier by a function vanishing on $\operatorname{sp}(U)=\widehat{G}$, thus $U(F)(I-P)=0$.

REMARK 2. The case of unbounded local multiplier $F$ often reduces to that of a bounded one, for example $(\hat{F}-\lambda)^{-1}$, if $\lambda$ does not belong to the closure of the range of $F$. Note that if $G=\boldsymbol{R}$ and $D$ is the generator of $U$, the spectral mapping theorem for $\hat{F}(D)=U(F)$ assumes the usual form $\sum(\hat{F}(D))=\hat{F}\left(\sum(D)\right)$.

Some functions may be of particular interest. If $\hat{F}(t)=e^{t}+e^{-t}$, the closure of $\hat{F}(D)$ is the inverse of the symmetric resolvent of $D$ [1]. If $\hat{F}(t)=e^{t}$, then $\hat{F}(D)$ is the analytic generator of $U$ [1]; in this case the spectral mapping theorem does not hold $[4,13]$, indeed either $\sum(\hat{F}(D))=\hat{F}\left(\sum(D)\right)$ or $\sum(\hat{F}(D))=C$. The second alternative being true for every nontrivial one parameter *automorphism group of a commutative $C^{*}$-algebra.

\section{REFERENCES}

1. J. Cioranescu and L. Zsido, Analytic generator for one parameter groups, Tôhoku Math. J., 28 (1976), 327.

2. F. Combes and C. Delaroches, Representations des groupes localement compacts et applications aux algebre d'operateurs Asterisque, 55.

3. A. Connes, Une classification des facteurs de tipe III, Ann. Sci. Ecole Norm. Sup., 6 (1973), 133.

4. A. Van Daele, On the spectrum of the analytic generator, Math. Scand, 37 (1975), 307.

5. D. E. Evans, On the spectrum of a one parameter strongly continuous representation, Math. Scand., 39 (1976), 80.

6. C. Fojas, Une application des distributions vectorielles a la theorie spectrale, Bull, Sci. Math. II, serie 84 (1960), 158

7. E. Hewitt and K. Ross, Abstract Harmonic Analysis, Springer-Verlag, Berlin, 1963.

8. T. Kato, Perturbation Theory for Linear Operators, Springer-Verlag, New York, 1966.

9. R. Longo, Some aspects of $C^{*}$-dynamics, in "Algèbres d'opérateurs et leur applications en physique mathèmatique" Colloques Internationaux du C.N.R.S. n ${ }^{\circ} 2741977$.

10. M. A. Naimark, Normed Rings, Noordhoff, Groningen, 1964.

11. W. Rudin, Fourier Analysis on Groups, Interscience, New York, 1962. 
12. R. Wyseur, An operational calculus for weak-continuous one parameter groups of weak-continuous isometries on a Banach space, Rev. Roum. Math. Pures et Appl., 23 (1978), 313.

13. L. Zsido, Spectral properties of the analytic generator and singular integrals, preprint, I. N. C. R. E. S. T., Bucharest.

Received July 10, 1979. Work supported in part by the Italian C.N.R. (G.N.A.F.A.).

UNIVERSita' Dell'aquila

L'AQUILA, ITALY

UNIVERSITA' DI ROMA

ROMA, ITALY

AND

I. N. C. R. E. S. T.

BUCHAREST, ROMANIA 


\section{PACIFIC JOURNAL OF MATHEMATICS}

\section{EDITORS}

DONALD BABBITT (Managing Editor)

University of California

Los Angeles, California 90024

Hugo RossI

University of Utah

Salt Lake City, UT 84112

C. C. Moore and Arthur Agus

University of California

Berkeley, CA 94720
J. DugundJI

Department of Mathematics University of Southern California Los Angeles, California 90007

R. FInN and J. Milgram Stanford University Stanford, California 94305

ASSOCIATE EDITORS
R. ARNES
E. F. BeCKenbach
B. H. NeumanN
F. WOLF
K. YoSHIDA

\section{SUPPORTING INSTITUTIONS}

UNIVERSITY OF ARIZONA

UNIVERSITY OF BRITISH COLUMBIA

CALIFORNIA INSTITUTE OF TECHNOLOGY

UNIVERSITY OF CALIFORNIA

MONTANA STATE UNIVERSITY

UNIVERSITY OF NEVADA, RENO

NEW MEXICO STATE UNIVERSITY

OREGON STATE UNIVERSITY
UNIVERSITY OF OREGON

UNIVERSITY OF SOUTHERN CALIFORNIA

STANFORD UNIVERSITY

UNIVERSITY OF HAWAII

UNIVERSITY OF TOKYO

UNIVERSITY OF UTAH

WASHINGTON STATE UNIVERSITY

UNIVERSITY OF WASHINGTON 


\section{Pacific Journal of Mathematics}

\section{Vol. 103, No. $1 \quad$ March, 1982}

Abdul Aziz, On the zeros of composite polynomials ..................

Salomon Benzaquen and Enrique M. Cabaña, The expected measure of the level sets of a regular stationary Gaussian process $\ldots \ldots \ldots \ldots \ldots$

Claudio D’Antoni, Roberto Longo and László Zsidó, A spectral mapping theorem for locally compact groups of operators $\ldots \ldots \ldots \ldots \ldots \ldots \ldots 17$

Ronald Dotzel, Semifree finite group actions on homotopy spheres ........ 25

Daniel H. Gottlieb, The Lefschetz number and Borsuk-Ulam theorems . . . . . 29

Shui-Hung Hou, On property $(Q)$ and other semicontinuity properties of

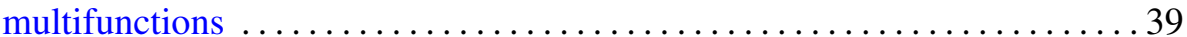

Kevin Mor McCrimmon, Compatible Peirce decompositions of Jordan triple systems

Mitsuru Nakai, Corona problem for Riemann surfaces of Parreau-Widom

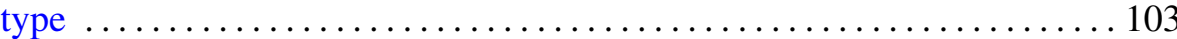

Jack Ray Porter and R. Grant Woods, Extensions of Hausdorff spaces . . . . 111

Milton Rosenberg, Quasi-isometric dilations of operator-valued measures

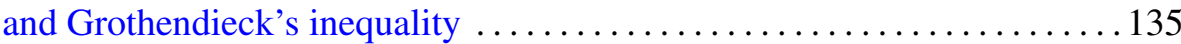

Joseph L. Taylor, A bigger Brauer group $\ldots \ldots \ldots \ldots \ldots \ldots \ldots \ldots \ldots \ldots$

Thomas Vogel, Symmetric unbounded liquid bridges . . . . . . . . . . . 205

Steve Wright, The splitting of operator algebras. II ............... 243 